\title{
Introduced birds in urban remnant vegetation: Does remnant size really matter?
}

\author{
MARK J. ANTOS, ${ }^{1,2}$ JAMES A. FITZSIMONS, ${ }^{1}$ GRANT C. PALMER ${ }^{1,3}$ AND \\ JOHN G. WHITE ${ }^{1 \star}$ \\ ${ }^{1}$ School of Life and Environmental Sciences, Deakin University, 221 Burwood Highway, Burwood, \\ Victoria, 3125, Australia (Email: john.white@deakin.edu.au), ${ }^{2}$ Birds Australia, Hawthorn East, \\ Victoria, ${ }^{3}$ Centre for Environmental Management, School of Science and Engineering, University of \\ Ballarat, Ballarat, Victoria, Australia
}

\begin{abstract}
Introduced birds are a pervasive and dominant element of urban ecosystems. We examined the richness and relative abundance of introduced bird species in small (1-5 ha) medium (6-15 ha) and large ( $>15 \mathrm{ha}$ ) remnants of native vegetation within an urban matrix. Transects were surveyed during breeding and non-breeding seasons. There was a significant relationship between introduced species richness and remnant size with larger remnants supporting more introduced species. There was no significant difference in relative abundance of introduced species in remnants of different sizes. Introduced species, as a proportion of the relative abundance of the total avifauna (native and introduced species), did not vary significantly between remnants of differing sizes. There were significant differences in the composition of introduced bird species between the different remnant sizes, with large remnants supporting significantly different assemblages than medium and small remnants. Other variables also have substantial effects on the abundance of introduced bird species. The lack of significant differences in abundance between remnant sizes suggests they were all equally susceptible to invasion. No patches in the urban matrix are likely to be unaffected by introduced species. The effective long-term control of introduced bird species is difficult and resources may be better spent managing habitat in a way which renders it less suitable for introduced species (e.g. reducing areas of disturbed ground and weed dominated areas).
\end{abstract}

Key words: introduced species, invasion, remnant size, urban avifauna

\section{INTRODUCTION}

Introduced birds are a pervasive and, in places, dominant element of urban ecosystems (White et al. 2005). Many of these introduced species are regarded as pests due to their real or perceived impact on agriculture and human structures, competition with native species for resources or their potential to spread weeds or hybridize with native species (Long 1981; Mullett 1996; Pell \& Tidemann 1997a,b; Lowe et al. 2000; Bomford \& Sinclair 2002; Hart 2002). Thirteen species of introduced birds occur in Melbourne's suburbs. These range from having serious pest status (e.g. common starling Sturnus vulgaris and common myna Acridotheres tristis) to moderate (e.g. spotted turtle-dove Streptopelia chinensis) and minor or non-pest status (e.g. European greenfinch Carduelis chloris) (Hart 2002).

Understanding the pattern of how introduced birds are distributed through the urban environment is critical to the development of management and conservation programmes. Soulé (1990) considered invasions

${ }^{\star}$ Corresponding author.

Accepted for publication December 2005 by introduced species and fragmentation as two of the most important factors impinging upon conservation into the future, while also indicating the need to improve our understanding of these factors.

The urban environment offers a unique area to examine landscape influences on biodiversity, given that there are often numerous small remnants of native vegetation isolated in a highly variable matrix. Recent studies by White et al. (2005) demonstrated that there were significant differences in the prevalence of introduced birds in remnants of native vegetation compared with the surrounding urban matrix. Within the urban matrix, differences in the prevalence of introduced birds were influenced by the type of streetscape vegetation (i.e. native vs. exotic), with exotic streetscapes having as much as $75 \%$ of their abundance of birds comprised of introduced species. Urban remnant vegetation contained introduced species but at a much reduced level when compared with the urban matrix.

The majority of research pertaining to introduced birds tends to occur at the regional or continental scale and focuses on either primarily natural or agricultural systems. Comparatively little work has been conducted on introduced bird species in urban areas 
(Chace \& Walsh 2006). While some studies have been conducted on individual bird species (e.g. Pell \& Tidemann 1994, 1997a,b; Wood 1995; Martin 1996), few have focused on the structure of introduced bird assemblages in Australia.

Remnants of native vegetation interspersed in urban areas provide habitat for native species in an otherwise largely unsuitable matrix (White et al. 2005). These remnants, however, are vulnerable to invasions of introduced bird species more typical of the urban matrix which may compromise their ability to support native bird species

Numerous hypotheses have been suggested as to what makes natural vegetation more or less susceptible to invasion by introduced species (e.g. Fox \& Fox 1986), but few have been tested. Fox and Fox (1986) proposed two models that may be important in relation to the susceptibility of urban remnants to invasions, these being (i) increased disturbance leads to higher levels of invasion, and (ii) habitats with high native species richness are less likely to be invaded. We propose that remnants of differing sizes in the urban environment could provide a good model to test these hypotheses. We predict that small urban remnants are more likely to be invaded than large remnants because they are more likely to suffer greater disturbance from the surrounding matrix due to increased edge effects and proximity to the matrix. Small remnants are also likely to have considerably reduced native species richness due to patch size and species richness relationships and thus would be expected to be more prone to invasion.

This paper aims to (i) investigate the pattern of introduced bird assemblages occurring in remnant vegetation in urban environments and the capacity of remnant vegetation to resist invasion, and (ii) investigate the characteristics of patches of remnant vegetation that determine their use by introduced species.

\section{METHODS}

Research was conducted in Melbourne, Victoria, Australia $\left(37^{\circ} 50^{\prime} \mathrm{S}, 44^{\circ} 58^{\prime} \mathrm{E}\right)$. A wide range of natural vegetation types occurred prior to settlement including forests, woodlands, heathlands, wetlands and grasslands. Remnants of many of these habitats may still be found within parks embedded in the urban matrix.

Study sites were located within the urban matrix of the Gippsland Plain IBRA subregion V5.1 (see Morgan 2001), mainly in the eastern and southeastern suburbs, within a $30-\mathrm{km}$ radius of the Melbourne Central Business District.

Remnants were selected based on size and availability and were at least $2 \mathrm{~km}$ apart. Remnants were divided into three size-classes - small (1-5 ha) $(n=15)$, medium (6-15 ha) $(n=14)$ and large $(>15$ ha) $(n=10)$.

\section{Bird surveys}

Transects of $1 \mathrm{~h}(200 \mathrm{~m} \times 50 \mathrm{~m})$ were used to assess species richness and relative abundance of introduced and native terrestrial birds (excluding aquatic species). One transect was used to survey small remnants, two transects were used in medium remnants and three transects were used in large remnants. Surveys were conducted during the non-breeding season (AprilJuly) of 2003 and breeding season (OctoberFebruary) of 2003/04. Each transect was surveyed four times in each season, on different days, between dawn and midday during favourable conditions (days of high wind or rain were avoided). All surveys were conducted by experienced bird observers (M.J.A., J.A.F., G.C.P.).

Each transect was surveyed on foot over a 10-min period and all birds seen and heard were recorded, including those flying above the canopy. The results from all transects within a remnant were pooled and averaged to produce measures of relative abundance.

\section{Habitat measures}

Quadrats were established to determine the number of trees per hectare, per cent canopy cover, the number of hollow-bearing trees and leaf litter depth and cover. Measures were also taken of different ground cover attributes, including the cover (\%) of bare ground, native grasses and herbs, exotic grasses and herbs and the number of logs. Measures were also taken of the cover (\%) of shrub and midstorey plants. A geographical information system (ArcView GIS 3.3, ESRI, Redlands, USA) was used to determine the size of each remnant patch, the proportion of the remnant that was made up of riparian vegetation, and the number of broad Ecological Vegetation Class (EVC) groupings represented in each remnant.

\section{Statistical analysis}

Analysis of variance (ANOVA) was used to determine whether remnant patch size had an effect on the richness of introduced and native woodland bird species, the proportion of the bird species richness that was introduced, the relative abundance of introduced and native woodland bird species, and the proportion of relative abundance that was introduced. StudentNewmann-Kuels (SNK) post-hoc tests were used to determine where significant differences were revealed by the ANOVA. 
To assess differences in the composition of introduced species populations between remnants of different size-classes, a similarity among sites matrix was developed using a Bray-Curtis index based on the mean number of each species observed in each remnant. Gross differences between remnants of different size-classes were compared using ANOSIM (analysis of similarity). The similarity percentage (SIMPER) procedure was used to identify those species that contributed most to the similarity of remnants within sizeclasses and dissimilarity between remnants in different size-classes (a $70 \%$ cut-off value was utilized). The ANOSIM and SIMPER procedures were conducted using the PRIMER software package (Clarke \& Warwick 1994).

To determine what drives the relative abundance of individual introduced species an information-theoretic modelling approach was taken, as described by Burnham and Anderson (2002). As this study had a small sample size and the data were not overdispersed, the second order Akaike information criterion corrected for small sample sizes $\left(\mathrm{AIC}_{\mathrm{c}}\right)$ was utilized. Models were developed for common mynas, common starlings, spotted turtle-doves and the common blackbird.

Generalized linear models assuming a Gaussian distribution were used to investigate the relationship between the relative abundance of species and predictor habitat variables. The predictor variables applied to all species were the area (ha) of the remnant $\left(\log _{10}\right)$ the number of broad EVC groupings represented in each remnant, the proportion of the remnant comprised of riparian vegetation, the percentage cover of exotic grasses, the overall percentage cover of ground vegetation, and the proportion of bare ground $\left(\log _{10}\right)$. Akaike differences $\left(\Delta_{i}\right)$ were used to determine the level of support for each model in the candidate set. Burnham and Anderson (2002) suggest that candidate models with Akaike differences less than 2 have substantial support. Akaike weights $\left(w_{i}\right)$ were additionally used to determine the evidence of support for each model.

Model averaging was used to give unconditional model variances when the Akaike weight suggested no model was clearly the best $\left(w_{i}<0.9\right)$ (Anderson et al.
2001). Weighted model averaging based on 1000 bootstrapped samples was used to reduce model selection bias. Hierarchical partitioning was used to determine the independent contribution of each predictor variable to the overall model.

Models were developed using $R$ statistical packages (Ihaka \& Gentleman 1996), using algorithms to calculate $\mathrm{AIC}_{\mathrm{c}}$, bootstrap frequencies, and model averaged estimates. Hierarchical partitioning was undertaken in $R$ using the hier.part package (Walsh \& Mac Nally 2003).

\section{RESULTS}

Species accumulation curves were developed to determine whether the number of survey visits to sites of differing sizes were sufficient to stabilize estimates of species richness. There was a significant difference in the number of species detected in the three different size-classes of remnants $\left(F_{2,288}=252.484, P<0.001\right)$. The number of species detected was also significantly affected by the number of visits to a site $\left(F_{7,288}=38.877, P<0.001\right)$. Overall, the number of species increased significantly with each visit (SNK $P<0.05)$ until the sixth visit, when the number of species stabilized for the final three visits (SNK $P>0.05)$, suggesting that six visits to each remnant would have been sufficient to survey the avifauna. There was also no significant interaction between remnant size and the number of visits to a site in regards to the number of species detected $\left(F_{14,288}=0.997\right.$, $P=0.456)$, indicating the methodology worked equally well over all size-classes of remnant.

Six introduced species were detected in the 39 remnants (Table 1). The spotted turtle-dove and the common blackbird were the most frequently recorded species being detected in 35 of the 39 remnants. The common myna occurred in 31 remnants and was detected in every large remnant (Table 1). The common starling was also widespread occurring in 21 of the remnants (Table 1). The song thrush and rock dove were rarely recorded and only encountered in four remnants.

Table 1. The occurrence (number of remnants the species occurred in) of introduced bird species in urban remnants of differing sizes, and the mean number of introduced species detected in the different remnant size-classes

\begin{tabular}{lcccc}
\hline Species & $1-5$ ha $(n=15)$ & $6-15$ ha $(n=14)$ & $>15$ ha $(n=10)$ & Total $(n=39)$ \\
\hline Spotted turtle-dove & 12 & 14 & 9 & 9 \\
Common blackbird & 12 & 14 & 10 & 35 \\
Common myna & 9 & 12 & 8 & 31 \\
Common starling & 8 & 5 & 2 & 21 \\
Rock dove & 2 & 0 & 2 & 4 \\
Song thrush & 0 & 2 & 4.00 & 4 \\
Mean number of species & 2.87 & 3.36 & 3.33 \\
\hline
\end{tabular}




\section{Species richness: context within remnant (transect level)}

Species richness was examined at the transect level by comparing the average number of species detected per transect within each remnant. This level of measurement provides an indication of the influence of transect context within the remnant on species richness. There was no significant difference in the number of introduced bird species detected per transect within remnants of the three different size-classes $\left(F_{2,36}=0.659, P=0.524\right)$. There was, however, a significant remnant patch size effect on the number of native bird species detected per transect $\left(F_{2,36}=6.455, P=0.004\right)$, with small patches having significantly fewer native bird species per transect than transects in medium and large remnants $(\mathrm{SNK}<0.05)$.

\section{Species richness: species area relationships (remnant level)}

Species area relationships were determined based on the total number of species detected in each remnant, combining the individual transects. There was a significant relationship between introduced species richness and remnant size $\left(F_{2,36}=5.156, P=0.011\right)$. Large remnants had significantly more introduced species (mean $=4.0$ species) than small remnants $($ mean $=2.9) \quad(\mathrm{SNK}<0.05)$. The number of introduced species in medium remnants was not significantly different to those in the small or large remnants $($ mean $=3.4)(\mathrm{SNK}>0.05)$.

There was a significant relationship between the richness of native woodland-associated bird species and the size of the remnant $\left(F_{2,36}=32.767, P<\right.$ $0.001)$. As remnants increased in size the number of native species increased significantly ( $\mathrm{SNK}<0.05)$. Small remnants contained 14.9 species, medium remnants contained 22.9 species and large remnants contained 31.6 native species on average.
The proportion of the total species in a remnant that were introduced differed significantly between remnants of different sizes $\left(F_{2,36}=7.103, P=0.003\right)$. Small remnants had a significantly higher proportion of introduced species (mean $=16.4 \%) \quad($ SNK $P<$ 0.05 ) than medium and large remnants (mean $=$ $12.9 \%$ and $11.3 \%$, respectively) (SNK $P>0.05$ ).

\section{Relative abundance}

There was no significant difference in the relative abundance of introduced species $\left(F_{2,36}=2.271\right.$, $P=0.118)$, woodland-associated native species $\left(F_{2,36}\right.$ $=0.930, P=0.404)$ and the total number of species $\left(F_{2,36}=1.069, P=0.354\right)$ in remnants of differing sizes. The proportion of the relative abundance of all birds that was comprised of introduced species did not differ significantly between remnants of differing sizes $\left(F_{2,36}=2.022, P=0.147\right)$, with $12.5 \%, 14.4 \%$ and $9.1 \%$ of the abundance being introduced species in the small, medium and large remnants, respectively.

\section{Composition}

There was a significant difference in the composition of introduced bird species between the different remnant sizes (ANOSIM), with none of the 999 random permutations exceeding the global $R$ statistic (0.155). There was no significant difference in the composition of introduced bird species between small- and medium-sized remnants $(P>0.05)$. However, there was a significant difference in the composition of introduced bird species between small and large remnants $(P=0.023)$ and medium and large remnants $(P=0.001)$.

The compositional differences between remnants of different sizes were a result of differences in the relative abundance of spotted turtle-doves, common blackbirds, common mynas and, to a lesser extent, common starlings (Table 2). These species were represented in

Table 2. Percentage contribution of species to similarities between small remnants (1-5 ha), medium remnants (6-15 ha) and large remnants ( $>15 \mathrm{ha}$ ), and to dissimilarities between the introduced avifauna of small (S) and medium (M) remnants (SvM), small and large (L) remnants (SvL) and medium and large remnants (MvL) based on Bray-Curtis indices (SIMPER)

\begin{tabular}{|c|c|c|c|c|c|c|}
\hline \multirow[b]{2}{*}{ Species } & \multicolumn{3}{|c|}{$\begin{array}{l}\text { Similarity } \\
(\% \text { contribution })\end{array}$} & \multicolumn{3}{|c|}{$\begin{array}{c}\text { Dissimilarity } \\
(\% \text { contribution })\end{array}$} \\
\hline & $S$ & M & $\mathrm{L}$ & SvM & SvL & MvL \\
\hline Spotted turtle-dove & 41.34 & 41.09 & 22.28 & 33.69 & 27.62 & 33.28 \\
\hline Common blackbird & 35.85 & 35.16 & 19.86 & 29.52 & 27.53 & 27.33 \\
\hline Common myna & 13.63 & 22.82 & 50.06 & 28.26 & 32.31 & 30.29 \\
\hline Common starling & & & & & 9.74 & \\
\hline Total contribution (\%) & 90.82 & 99.07 & 92.19 & 91.47 & 97.20 & 90.90 \\
\hline
\end{tabular}


all the different remnant size groupings but their relative abundance varied considerably (Fig. 1).

\section{Individual species responses}

Akaike information criteria models were conducted to understand the relative importance of remnant size versus other environmental variables in influencing the relative abundance of the most common introduced species.

Seven models were produced with substantial support based on AICc differences for spotted turtle-dove abundance. These models suggested that remnant area, exotic grass cover, the amount of bare ground and riparian vegetation and the number of EVC groupings in a remnant influenced the abundance of spotted turtle-doves (Table 3). Low AIC weights suggest that there is considerable model uncertainty and hence model averaging was conducted (Table 3 ). Based on model averaging and hierarchical partitioning, the main contributing factor $(40.5 \%)$ to spotted turtle-dove abundance is a negative relationship with remnant area (Table 4). Spotted turtle-dove abundance also showed a negative relationship with the amount of bare ground ( $17.1 \%$ contribution). The cover of exotic grasses $(19.5 \%$ contribution) and the amount of riparian vegetation ( $11.7 \%$ contribution) were positively related with spotted turtle-dove abundance (Table 4).

Common blackbird abundance was explained by five models which had substantial support based on AICc (Table 3). While no model was better than the others, remnant area, the number of EVC groupings, the amount of riparian vegetation and the overall cover

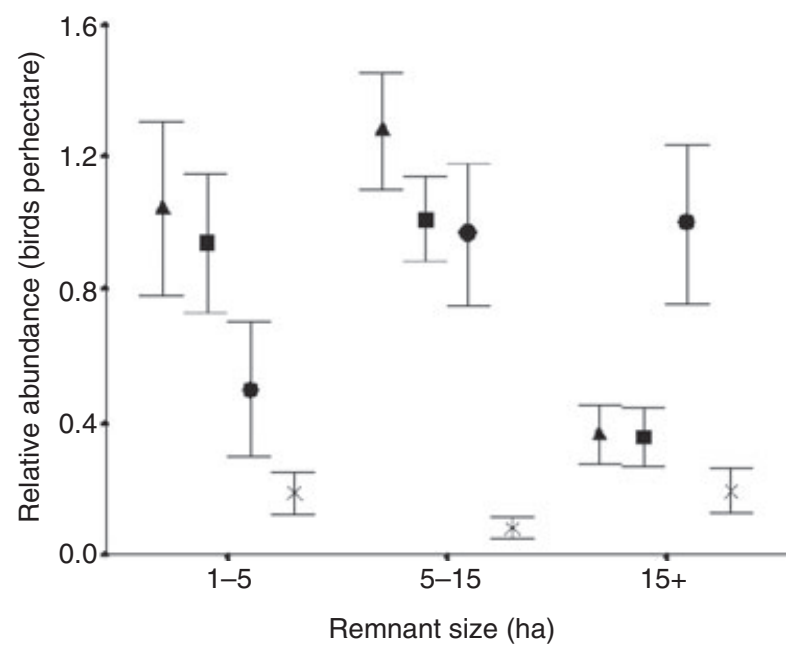

Fig. 1. The relative abundance (mean $\pm 1 \mathrm{SE}$ ) of introduced bird species in urban remnants of differing sizes. (A)


myna, and $(x)$ common starling. of ground vegetation were involved in the strongest models. Hierarchical partitioning and model averaging suggest that common blackbird abundance is mainly related to remnant size and the number of EVC groupings. Remnant area is negatively related with the abundance of common blackbirds ( $23.5 \%$ contribution) as is the number of EVC groupings (17.2\% contribution) (Table 4).

Three models were produced for the abundance of common mynas with substantial support (Table 3), but low AIC weight values suggest a great deal of model uncertainty. The main variables used in the substantially supported models were the number of EVC groupings, the amount of riparian vegetation, the amount of exotic grasses and the size of the remnant patch. Hierarchical partitioning and model averaging was conducted as no individual model was supported by AICc. Common myna abundance is positively related with the amount of riparian vegetation $(23 \%$ contribution), the cover of exotic grasses (18.6\%) and the number of EVC groupings (13.2\%) (Table 4). Remnant area had a limited positive relationship with common myna abundance, but was not a highly important contributing variable.

The abundance of common starlings was explained by four models with substantial support. While there is considerable model uncertainty, the main factors used in the best models were the number of EVC groupings, remnant area and the cover of exotic grasses (Table 3). As no clear best model is produced, model averaging and hierarchical partitioning were conducted. The abundance of common starlings was negatively related to the number of EVC groupings ( $87.7 \%$ contribution) and positively related with the cover of exotic grasses $(23.3 \%)$ (Table 4 ). Remnant area was also negatively related with the abundance of common starlings $(12.2 \%)$ (Table 4$)$.

\section{DISCUSSION}

Remnant size had a significant effect on the richness and composition of introduced bird assemblages, but no significant effect on the relative abundance of introduced bird assemblages or the proportion of the abundance of all species. All remnants, irrespective of size, supported a similar baseline abundance of introduced birds. This abundance was relatively low when compared with those recorded in the surrounding urban matrix (see White et al. 2005).

Differences in introduced bird assemblages and the differing responses of individual species are a reflection of the broad range of foraging and nesting ecologies as well as habitat preferences of these species. For instance, the spotted turtle-dove is a granivore which requires frequent access to water, hence the preference for areas with high exotic grass cover and riparian 
Table 3. AICc based model selection for spotted turtle-dove, common blackbird, common starling and common myna based on estimates of relative abundance

\begin{tabular}{|c|c|c|c|c|c|c|c|}
\hline Species & Model $^{\star}$ & $\log (\mathrm{L})$ & $K$ & $\mathrm{AICc}$ & $\Delta \mathrm{AIC}$ & $w_{i}$ & $i$ \\
\hline \multirow[t]{10}{*}{ Spotted turtle-dove } & area & -46.4 & 3 & 99.500 & 0.000 & 0.085 & 0.143 \\
\hline & grass & -46.6 & 3 & 100.000 & 0.441 & 0.068 & 0.097 \\
\hline & area, bare & -45.5 & 4 & 100.300 & 0.719 & 0.059 & 0.057 \\
\hline & bare & -46.9 & 3 & 100.500 & 0.926 & 0.053 & 0.105 \\
\hline & area, rip & -45.6 & 4 & 100.500 & 0.932 & 0.053 & 0.086 \\
\hline & area, grass & -45.7 & 4 & 100.600 & 1.076 & 0.049 & 0.036 \\
\hline & evc & -47.3 & 3 & 101.300 & 1.771 & 0.035 & 0.024 \\
\hline & area, rip, bare & -44.9 & 5 & 101.700 & 2.144 & 0.029 & 0.040 \\
\hline & rip & -47.5 & 3 & 101.700 & 2.157 & 0.029 & 0.016 \\
\hline & ogv & -47.5 & 3 & 101.700 & 2.196 & 0.028 & 0.019 \\
\hline \multirow[t]{10}{*}{ Common blackbird } & area & -36.9 & 3 & 80.400 & 0.000 & 0.115 & 0.229 \\
\hline & evc & -37.1 & 3 & 80.900 & 0.473 & 0.091 & 0.151 \\
\hline & rip & -37.7 & 3 & 82.000 & 1.541 & 0.053 & 0.029 \\
\hline & ogv & -37.8 & 3 & 82.200 & 1.786 & 0.047 & 0.099 \\
\hline & area, ogv & -36.6 & 4 & 82.400 & 1.996 & 0.042 & 0.042 \\
\hline & grass & -37.9 & 3 & 82.600 & 2.112 & 0.040 & 0.103 \\
\hline & area, evc & -36.7 & 4 & 82.700 & 2.209 & 0.038 & 0.002 \\
\hline & bare & -38.0 & 3 & 82.700 & 2.227 & 0.038 & 0.057 \\
\hline & evc, ogv & -36.8 & 4 & 82.800 & 2.364 & 0.035 & 0.018 \\
\hline & area, bare & -36.8 & 4 & 82.800 & 2.388 & 0.035 & 0.074 \\
\hline \multirow[t]{10}{*}{ Common myna } & evc, rip, grass & -38.2 & 5 & 88.150 & 0.000 & 0.157 & 0.125 \\
\hline & rip, grass & -39.8 & 4 & 88.760 & 0.606 & 0.116 & 0.147 \\
\hline & area, rip, grass & -39.0 & 5 & 89.790 & 1.640 & 0.069 & 0.038 \\
\hline & evc, rip, grass, bare & -37.9 & 6 & 90.430 & 2.278 & 0.050 & 0.032 \\
\hline & rip, evc, bare & -39.4 & 5 & 90.680 & 2.530 & 0.044 & 0.087 \\
\hline & area, evc, rip, grass & -38.1 & 6 & 90.820 & 2.675 & 0.041 & 0.025 \\
\hline & evc, rip, grass, ogv & -38.1 & 6 & 90.900 & 2.747 & 0.040 & 0.022 \\
\hline & evc, grass & -40.9 & 4 & 91.060 & 2.911 & 0.037 & 0.034 \\
\hline & $\operatorname{rip}$ & -42.3 & 3 & 91.220 & 3.070 & 0.034 & 0.042 \\
\hline & evc, rip & -41.0 & 4 & 91.230 & 3.081 & 0.034 & 0.069 \\
\hline \multirow[t]{10}{*}{ Common starling } & evc & 10.0 & 3 & -13.303 & 0.000 & 0.125 & 0.167 \\
\hline & area, evc & 11.1 & 4 & -12.998 & 0.305 & 0.107 & 0.157 \\
\hline & evc, grass & 11.0 & 4 & -12.792 & 0.511 & 0.097 & 0.102 \\
\hline & area, evc, grass & 11.9 & 5 & -11.886 & 1.417 & 0.062 & 0.058 \\
\hline & evc, rip & 10.2 & 4 & -11.296 & 2.007 & 0.046 & 0.011 \\
\hline & evc, ogv & 10.1 & 4 & -10.985 & 2.318 & 0.039 & 0.015 \\
\hline & evc, bare & 10.0 & 4 & -10.853 & 2.450 & 0.037 & 0.004 \\
\hline & evc, rip, ogv & 11.3 & 5 & -10.688 & 2.615 & 0.034 & 0.030 \\
\hline & area, evc, bare & 11.2 & 5 & -10.513 & 2.791 & 0.031 & 0.006 \\
\hline & area, evc, ogv & 11.2 & 5 & -10.485 & 2.818 & 0.031 & 0.013 \\
\hline
\end{tabular}

Values represent the maximized $\log$-likelihood $(\log (\mathrm{L}))$, number of parameters $(K)$, Akaike information criterion corrected for small sample sizes (AICc), AIC differences ( $\triangle \mathrm{AIC}$ ), Akaike weights $\left(w_{i}\right)$ and bootstrap selection frequencies $(i)$ for the best models. The 10 highest ranked models based on AICc ranking are shown in descending order.

${ }^{\star}$ Model variables include: area $=$ area of remnant in hectares $($ logged), evc $=$ number of Ecological Vegetation Class broad groupings in each remnant, rip $=$ the proportion of the remnant that is comprised of riparian vegetation, grass = cover of exotic grasses $(\%)$, ogv $=$ the overall cover of ground vegetation $(\%)$, bare $=$ the proportion of the ground that has no ground cover (logged).

vegetation. Similarly, the common blackbird requires dense, low cover and showed a positive response to ground cover vegetation, while the common starling requires open areas for foraging and showed a positive response to exotic grass cover.

Introduced bird species are often lumped together and treated as a group by many papers (e.g. Green 1986; Parsons et al. 2003; Catterall 2004; White et al. 2005), yet our study has demonstrated that individual species show different responses to different variables. Some attributes such as managed exotic grass cover, however, seem to play an important role in determining the presence of most exotic species (e.g. Green 1986).

Our study did not support the hypothesis that urban remnants with high native species richness are more resistant to invasion by introduced species. In terms of introduced bird species richness, the results of our study reject this hypothesis, with more native and 
Table 4. Model averaged coefficients, unconditional and conditional standard errors, for all variables in introduced bird species models

Standard errors

\begin{tabular}{|c|c|c|c|c|c|}
\hline & Variables ${ }^{\star}$ & Coefficient & Unconditional & Conditional & Contribution \\
\hline \multirow[t]{6}{*}{ Spotted turtle-dove } & area & -0.230 & 0.233 & 0.322 & 40.460 \\
\hline & evc & -0.008 & 0.057 & 0.143 & 5.160 \\
\hline & rip & 0.199 & 0.427 & 0.630 & 11.720 \\
\hline & grass & 0.004 & 0.006 & 0.010 & 19.480 \\
\hline & ogv & 0.000 & 0.006 & 0.013 & 1.960 \\
\hline & bare & -0.160 & 0.316 & 0.489 & 17.120 \\
\hline \multirow[t]{6}{*}{ Common blackbird } & area & -0.117 & 0.156 & 0.262 & 23.539 \\
\hline & evc & -0.024 & 0.057 & 0.116 & 17.249 \\
\hline & rip & -0.018 & 0.128 & 0.512 & 6.113 \\
\hline & grass & 0.000 & 0.003 & 0.008 & 0.981 \\
\hline & ogv & 0.001 & 0.005 & 0.011 & 9.357 \\
\hline & bare & -0.016 & 0.177 & 0.398 & 0.537 \\
\hline \multirow[t]{6}{*}{ Common myna } & area & 0.088 & 0.198 & 0.271 & 7.633 \\
\hline & evc & 0.079 & 0.098 & 0.120 & 13.187 \\
\hline & rip & 0.959 & 0.511 & 0.529 & 23.002 \\
\hline & grass & 0.012 & 0.007 & 0.008 & 18.575 \\
\hline & ogv & 0.001 & 0.005 & 0.011 & 3.970 \\
\hline & bare & 0.057 & 0.215 & 0.411 & 1.294 \\
\hline \multirow[t]{6}{*}{ Common starling } & area & -0.029 & 0.056 & 0.076 & 12.202 \\
\hline & evc & 0.047 & 0.032 & 0.034 & 87.781 \\
\hline & rip & -0.007 & 0.061 & 0.148 & 1.877 \\
\hline & grass & 0.001 & 0.002 & 0.002 & 23.323 \\
\hline & ogv & 0.000 & 0.001 & 0.003 & 1.677 \\
\hline & bare & 0.001 & 0.034 & 0.115 & 0.751 \\
\hline
\end{tabular}

Results from hierarchical partitioning are shown, with the percentage independent contribution.

${ }^{\star}$ Model variables include: area = area of remnant in hectares (logged), evc = number of Ecological Vegetation Class groupings in each remnant, rip = the proportion of the remnant that is comprised of riparian vegetation, grass = cover of exotic grasses $(\%)$, ogv $=$ the overall cover of ground vegetation $(\%)$, bare $=$ the proportion of the ground that has no ground cover $($ logged $)$.

introduced species present in larger remnants. That the abundance of introduced bird species does not change according to the size of urban remnants further leading us to reject the hypothesis that disturbed small remnants are more likely to be invaded by introduced species.

An explanation for the comparatively low abundance of introduced species in remnants may be attributed to the fact that many of these species have existed overseas in human-dominated landscapes and thus may show a degree of pre-adaptation for disturbed environments over less disturbed native Australian ecosystems. It is important to note that seven introduced species present in Melbourne were not detected in remnants during this study. For example, the house sparrow Passer domesticus, which is one of the most reliant species on human-created environments, was not detected in any of the remnants, despite being a relatively common bird throughout urban Melbourne. Other commensal and widespread species, such as the rock dove, were also rarely recorded within remnants. This indicates that to some extent remnant vegetation of and size can be resistant to at least some of the introduced species in the urban landscape.

\section{Implications for management}

This research has shown that, for the most part, introduced species are only present at low, abundances in urban remnants. Whether such abundances pose threats to native biodiversity or other remnant values requires further research but would depend on the individual species. The case for controlling such species, which are often perceived as pests, needs to be considered in light of the impacts introduced bird species pose at these abundances (see Braysher 1993). Population control is likely to be cost- and labourintensive, short-term, and unpalatable with some members of community, and have uncertain outcomes (Temple 1990; Van Vuren \& Smallwood 1996; Johnston \& Marks 1997; Bomford \& Sinclair 2002; Tidemann 2002). Habitat manipulation provides an alternative management approach that may be more effective in reducing competitive interactions between introduced and native species in urban remnants. For example, remnant management could focus on improving the quality of vegetation for native species by reducing the availability of exotic grass cover and replacing it with native ground vegetation. 
There may be instances when control is required, especially where cavity-nesting species such as the common myna and the common starling compete for nest sites with native species (see Pell \& Tidemann 1997a,b). The effect of the introduced species needs to be quantified, however, to determine the potential benefit relative to cost of any such control programme.

\section{ACKNOWLEDGEMENTS}

We thank Amber Cameron and Christina Wilson for collecting vegetation data used in this paper. We are grateful to the Parks Victoria Research Partners Scheme for generous funding of the research. We thank Harry Recher and an anonymous referee for helpful comments on an earlier draft of the manuscript.

\section{REFERENCES}

Anderson D. R., Link W. A., Johnson D. H. \& Burnham K. P. (2001) Suggestions for presenting the results of data analyses. F. Wildl. Manag. 65, 373-8.

Bomford M. \& Sinclair R. (2002) Australian research on bird pests: impact, management and future directions. Emu 102, $29-45$.

Braysher M. (1993) Managing Vertebrate Pests: Principles and Strategies. Australian Government Publishing Service, Canberra.

Burnham K. P. \& Anderson D. R. (2002) Model Selection and Multimodel Inference: A Practical Information-Theoretical Approach, 2nd edn. Springer-Verlag, New York.

Catterall C. P. (2004) Birds, garden plants and suburban bushlots: where good intentions meet unexpected outcomes. In: Urban Wildlife: More Than Meets the Eye (eds D. Lunney \& S. Burgan) pp. 21-31. Royal Zoological Society of New South Wales, Mosman.

Chace J. F. \& Walsh J. J. (2006) Urban effects on native avifauna: a review. Landsc. Urban Plan 75, 46-69.

Clarke K. R. \& Warwick R. M. (1994) Change in Marine Communities: An Approach to Statistical Analysis and Interpretation. Plymouth Marine Laboratory: Plymouth.

Fox M. D. \& Fox B. J. (1986) The susceptibility of natural communities to invasion. In: Ecology of Biological Invasions: An Australian Perspective (eds R. H. Groves \& J. J. Burdon) pp. 57-66. Australian Academy of Science, Canberra.

Green R. J. (1986) Native and exotic birds in the suburban habitat. In: The Dynamic Partnership: Birds and Plants in Southern Australia (eds H. Ford \& D. C. Paton) pp. 130-9. The Flora and Fauna of South Australia Handbooks Committee, Adelaide.

Hart Q. (2002) Science for Decision Makers: Managing Pest Animals in Australia. Bureau of Rural Sciences, Canberra.

Ihaka R. \& Gentleman R. (1996) R.: a language for data analysis and graphics. F. Comp. Graph. Statist. 5, 299-314.
Johnston M. J. \& Marks C. A. (1997) Attitudinal Survey on Vertebrate Pest Management in Victoria. Vertebrate Pest Research Unit, Department of Natural Resources and Environment, Melbourne.

Long J. L. (1981) Introduced Birds of the World. Reed, Sydney.

Lowe S., Browne M., Boudjelas S. \& De Poorter M. (2000) 100 of the World's Worst Invasive Alien Species: A selection from the Global Invasive Species Database. Invasive Species Specialist Group of the IUCN Species Survival Commission. [Cited 28 Dec 2004.] Available from URL: http://www.issg.org/ booklet.pdf

Martin W. K. (1996) The current and potential distribution of the common myna Acridotheres tristis in Australia. Emu 96, 166-73.

Morgan G. (2001) Landscape Health in Australia: A Rapid Assessment of the Relative Condition of Australia's Bioregions and Subregions. Environment Australia and National Land \& Water Resources Audit, Canberra.

Mullett T. L. (1996) Ecological aspects of Sweet Pittosporum (Pittosporum undulatum): implications for control and management. Proceedings of the Eleventh Australian Weeds Conference; 28 Dec 2004, Weed Science E Technology, Melbourne, pp. 489-92.

Parsons H., French K. \& Major R. E. (2003) The influence of remnant bushland on the composition of suburban bird assemblages in Australia. Landsc. Urban Plan. 66, 43-56.

Pell A. S. \& Tidemann C. R. (1994) Population densities of common mynas, common starlings, crimson rosellas and eastern rosellas in Oakey Hill Nature Park and adjacent suburbs. Canberra Bird Notes 19, 58-66.

Pell A. S. \& Tidemann C. R. (1997a) The ecology of the common myna (Acridotheres tristis) in urban nature reserves in the Australian Capital Territory. Emu 97, 141-9.

Pell A. S. \& Tidemann C. R. (1997b) The impact of two exotic hollow-nesting birds on two native parrots in savannah and woodland in eastern Australia. Biol. Conserv. 79, 145-53.

Soulé M. M. (1990) The onslaught of alien species, and other challenges for the coming decades. Conser. Biol. 4, 233-9.

Temple S. A. (1990) The nasty necessity: eradicating exotics. Conser. Biol. 4, 113-5.

Tidemann C. R. (2002) Mitigation of the Impacts of Mynas on Biodiversity and Public Amenity. School of Resources, Environment \& Society, Australian National University Canberra. [Cited 28 Dec 2004.] Available from URL: http:/ /sres.anu.edu.au/associated/myna/minimise_files/ Myna_Mitigation_PhaseI.pdf

Van Vuren D. \& Smallwood K. S. (1996) Ecological management of vertebrate pests in agricultural systems. Biol. Agric. Hort. 13, 36-62.

Walsh C. \& Mac Nally R. (2003) The hier.part package. Hierarchical Partitioning. R. project for statistical computing. [Cited 28 Dec 2004.] Available from URL: http://cran. r-project.org/

White J. G., Antos M. J., Fitzsimons J. A. \& Palmer G. C. (2005) Non-uniform bird assemblages in urban environments: the influence of streetscape vegetation. Landsc. Urban Plan. 71, 123-35.

Wood K. A. (1995) Roost abundance and density of the common myna and common starling at Wollongong, New South Wales. Aust. Bird Watcher 16, 58-67. 